\title{
Determinants of left ventricular ejection fraction and a novel method to improve its assessment of myocardial contractility
}

\author{
Manuel Ignacio Monge García ${ }^{*}$, Zhongping Jian², Jos J. Settels², Charles Hunley ${ }^{3}$, Maurizio Cecconi ${ }^{4}$, \\ Feras Hatib² and Michael R. Pinsky ${ }^{5}$
}

\begin{abstract}
Background: The aim of this study was to quantify the impact of different cardiovascular factors on left ventricular ejection fraction (LVEF) and test a novel LVEF calculation considering these factors.

Results: 10 pigs were studied. The experimental protocol consisted of sequentially changing afterload, preload and contractility. LV pressure-volume (PV) loops and peripheral arterial pressure were obtained before and after each intervention. LVEF was calculated as stroke volume (SV)/end-diastolic volume (EDV). We studied global cardiac function variables: LV end-systolic elastance (Ees), effective arterial elastance (Ea), end-diastolic volume and heart rate. Diastolic function was evaluated by means of the ventricular relaxation time $(\tau)$ and ventricular stiffness constant $(\beta)$ obtained from the end-diastolic PV relationship. Ventriculo-arterial coupling (VAC), an index of cardiovascular performance, was calculated as Ea/Ees. LV mechanical efficiency (LVeff) was calculated as the ratio of stroke work to LV pressure-volume area. A linear mixed model was used to determine the impact of cardiac factors (Ees, Ea, EDV and heart rate), VAC and LVeff on LVEF during all experimental conditions. LVEF was mainly related to Ees and Ea. There was a strong relationship between LVEF and both VAC and LVeff $\left(r^{2}=0.69\right.$ and $r^{2}=0.94$, respectively). The relationship between LVEF and Ees was good $\left(r^{2}=0.43\right)$. Adjusting LVEF to afterload ( $\left.L V E F E A=E F \times \sqrt{E a}\right)$ performed better for estimating Ees $\left(r^{2}=0.75\right)$ and improved the tracking of LV contractility changes, even when a peripheral Ea was used as surrogate $\left(\mathrm{Ea}=\right.$ radial $\left.\mathrm{MAP} / \mathrm{SV} ; \mathrm{r}^{2}=0.73\right)$.
\end{abstract}

Conclusions: LVEF was mainly affected by contractility and afterload changes and was strongly related to VAC and LVeff. An adjustment to LVEF that considers the impact of afterload provided a better assessment of LV contractility.

Keywords: Ejection fraction, Systolic function, Diastolic function, Contractility, Ventriculo-arterial coupling, Ventricular efficiency, Afterload, Preload, Arterial elastance

\section{Background}

Currently, the most commonly method for the assessment of left ventricular (LV) systolic function in clinical practice is based on estimates of LV ejection fraction (LVEF) by either direct measures of ventricular volumes during cardiac catheterization or using two-dimensional echocardiography. LVEF characterizes LV performance by expressing ventricular ejection (stroke volume, SV)

\footnotetext{
*Correspondence: ignaciomonge@gmail.com

1 Unidad de Cuidados Intensivos, Hospital Universitario SAS de Jerez, C/ Circunvalación, s/n, 11407 Jerez de la Frontera, Spain

Full list of author information is available at the end of the article
}

as a fraction of the preload (end-diastolic volume, EDV). LVEF is a strong independent predictor of mortality in patients with both heart failure and acute myocardial infarct [1-3] and plays an essential role in therapeutic decision making $[4,5]$. However, LVEF is an inaccurate marker of LV intrinsic contractility $[6,7]$, because it is strongly influenced by LV loading conditions. For example, LVEF's ability to detect LV systolic dysfunction has been recently questioned in septic shock patients $[8,9]$. A normal LVEF associated with a severely depressed arterial tone, as described in sepsis, can be observed even in the presence of a severely impaired LV intrinsic 
contractility [9]. Furthermore, changes in LVEF following the introduction of vasoconstrictors have been questioned to reflect actual changes in LV contractility, but rather to unmask prior LV dysfunction when LV afterload has been corrected $[9,10]$.

Since LVEF must represent the interaction of several cardiovascular factors, we aimed to independently assess the contribution of different determinants affecting LVEF and also to test a novel LVEF calculation for estimating actual LV contractility considering the impact of these determinants. We performed this analysis on hemodynamic data also reported for another study addressing different issues [11]. This study is a continuation of our previous works about central and peripheral hemodynamics $[11,12]$ and represents a physiological exploration of the determinants of LVEF without any commercial interest.

\section{Methods}

\section{Animals}

The study protocol was approved by the Institutional Animal Care and Use Committee (IACUC) at the Edwards Research Center and performed in accordance with the USDA Animal Welfare Act regulations (AWArs), and the Guide for the Care and Use of Laboratory Animals (ILAR, NAP, Washington, DC, 2010, 8th edition).

Ten anaesthetized and mechanically ventilated adult Yorkshire pigs weighting $81 \pm 6 \mathrm{~kg}$ were studied. Animals were premedicated with intramuscular telazol (4.4 $\left.\mathrm{mg} \mathrm{kg}^{-1}\right)$, ketamine $\left(2.2 \mathrm{mg} \mathrm{kg}{ }^{-1}\right)$ and xylazine $\left(1.1 \mathrm{mg} \mathrm{kg}^{-1}\right)$. They were orally intubated and mechanically ventilated in a volume-controlled mode $\left(\mathrm{FIO}_{2}\right.$ $60-80 \%$, tidal volume $10 \mathrm{ml} \mathrm{kg} \mathrm{kg}^{-1}$ at respiratory rate 13-15 cycles $\mathrm{min}^{-1}$ ). Following endotracheal intubation, general anesthesia was maintained with isoflurane 1.5$2.5 \%$ and a mixture of oxygen, air and/or nitrous oxide. Fluid maintenance was provided by an intravenous infusion of Ringer's lactate solution at $2-4 \mathrm{ml} \mathrm{kg}^{-1} \mathrm{~h}^{-1}$. Rectal temperature was monitored and kept between 36 and $37^{\circ} \mathrm{C}$ using a heating pad. Animal anesthesia was monitored and recorded approximately every $15 \mathrm{~min}$ for the duration of the experimentation.

Instantaneous LV pressure-volume (PV) measurements were obtained from a dual-field conductance catheter and a high-fidelity pressure sensor (CA71083PL, CD Leycom, Zoetermeer, the Netherlands) connected to a PV signal processor (Inca ${ }^{\circledR}$, CD Leycom). The catheter tip was positioned in the LV apex and the correct placement confirmed by fluoroscopy and the examination of the segmental LV PV loops. Radial pressure was continuously recorded with a fluid-filled pressure transducer (FloTracIQ sensor, Edwards Lifesciences, Irvine, CA, USA) using the EV1000 monitor (Edwards Lifesciences).

\section{Data collection and analysis}

Volume signal calibration consisted of the determination of cardiac output ( $\mathrm{CO}$ ) by the standard thermodilution method and correction for parallel conductance by the hypertonic saline method [13, 14]. Calibration was performed before starting the experimental protocol and repeated after the fluid bolus stage. LV pressure-volume signals were recorded at $250 \mathrm{~Hz}$ sampling rate, filtered using a $25 \mathrm{~Hz}$ low-pass filter and analyzed in a dedicated software (Conduct NT, version 3.18.1, CD Leycom).

Global cardiac function, ventriculo-arterial coupling and LV mechanical efficiency Before and after each experimental stage, three partial occlusions of the inferior vena cava (IVC) were performed using a Fogarty balloon during apnea. This procedure was repeated if ectopic beats were detected. LV end-systolic elastance (Ees), a load-independent measure of LV contractility [15], was determined as the slope of the end-systolic pressure-volume relationship during the first $10 \mathrm{~s}$ of the IVC occlusion, calculated from the linear regression analysis of the maximal elastance points on each cardiac cycle, defined as $E(t)=P(t) / V(t)-V_{0}$, where $V_{0}$ is volume-axis intercept or the LV unstressed volume [13]. End-systolic pressure (Pes), stroke volume (SV), CO, EDV and end-systolic volume (ESV), end-diastolic pressure, LVEF, effective arterial elastance $(\mathrm{Ea}=\mathrm{Pes} /$ SV, a lumped parameter of LV afterload) [16] and radial arterial pressure were calculated from 3 to 5 beats in steady-state conditions during apnea just before the IVC occlusion. Ventriculo-arterial coupling (VAC) was computed as the ratio of Ea and Ees [17]. LV pressurevolume area (PVA) represents the total LV mechanical energy and was determined as the sum of stroke work (SW, or the integrated area within each LV PV loop) and potential energy $(\mathrm{PE})$, where $\mathrm{PE}=\operatorname{Pes} \times\left(\mathrm{ESV}-V_{0}\right) / 2$. The ratio of SW/PVA, expressed as a percentage, represents therefore the LV mechanical efficiency (LVeff) [18]. As Pes can be estimated from peripheral mean arterial pressure (MAP), a peripheral Ea was also calculated as: $\mathrm{Ea}_{\text {periph }}=$ radial MAP/SV [12].

$L V$ diastolic function We calculated the LV chamber stiffness, a load-independent index of diastolic function representing the passive viscoelastic properties of the $L V$, from the exponential curve fit of the end-diastolic pressure-volume relationship (EDPVR) during the IVC maneuver, as $\mathrm{EDP}=\alpha \times e^{\beta \times \mathrm{EDV}}$, being $\alpha$ the curve fitting constant and $\beta$ the LV chamber stiffness constant [19]. The time constant of the isovolumetric LV active relaxation $(\tau)$ was calculated as the time from $\mathrm{d} P / \mathrm{d} t_{\text {min }}$ until LV pressure reaches half value at the $\mathrm{d} P / \mathrm{d} t_{\min }[19$, 20]. End-diastole was defined as the moment on the peak of the $\mathrm{R}$ wave on the EKG. 


\section{Experimental protocol}

Before starting the protocol, animals received fluid resuscitation (Voluven ${ }^{\circledR}, 130 / 0.4$, Fresenius Kabi Deutschland $\mathrm{GmbH}$, Bad Homburg, German) until no significant change in $\mathrm{CO}$ was observed. Then they were allowed to stabilize for at least $10 \mathrm{~min}$ (heart rate and MAP variation $<5 \%$ ). The study protocol consisted of three consecutive stages with up and down interventions each: changes in afterload (phenylephrine and nitroprusside), preload (bleeding and fluid bolus), and contractility (esmolol and dobutamine). The experiment started with the afterload interventions: Animals were treated with sodium nitroprusside (100-200 $\mathrm{mg} \mathrm{kg}^{-1} \mathrm{~min}^{-1}$ ) to decrease MAP to $40 \%$ from baseline (but not below $50 \mathrm{mmHg}$ for allowing an adequate hemodynamic tolerance during the IVC occlusions), followed by recovery to baseline status. Then, they were treated with a phenylephrine infusion (30-120 $\mathrm{mg} \mathrm{kg}^{-1} \mathrm{~min}^{-1}$ ) to increase MAP by $40 \% \mathrm{mmHg}$ from baseline and were allowed to recover. Subsequently, for preload interventions, the animals were submitted to a stepwise bleeding of $12 \mathrm{ml} \mathrm{kg}^{-1}\left(50 \mathrm{ml} \mathrm{min}^{-1}\right)$ and the blood stored into a heparinized sterile bag. Then, the blood was reinfused at $50 \mathrm{ml} \mathrm{min}$, and a fluid bolus of $10 \mathrm{ml} \mathrm{kg}{ }^{-1}$ of colloid in $5 \mathrm{~min}$ was infused. After the fluid administration, the contractility interventions followed: An esmolol infusion was introduced at $50 \mu \mathrm{g} \mathrm{kg}{ }^{-1} \mathrm{~min}^{-1}$ and increased until decreasing $\mathrm{LV} \mathrm{d} P / \mathrm{d} t_{\max }$ by $50 \%$ from its previous value (maximal dose: $200 \mu \mathrm{g} \mathrm{kg} \mathrm{km}^{-1}$ ). Then, the esmolol infusion was stopped and, after a period of recovery, the animals were treated with dobutamine $\left(5 \mu \mathrm{g} \mathrm{kg}^{-1} \mathrm{~min}^{-1}\right)$ to increase $\mathrm{LV} \mathrm{d} P / \mathrm{d} t_{\max }$ by $50 \%$. LV PV loops and radial arterial pressure were obtained before and after each intervention.

\section{Statistical analysis}

Data are expressed as the mean $\pm \mathrm{SD}$, unless otherwise stated. Data normality was checked by the Shapiro-Wilk test. A linear mixed-effects model analysis was used to identify the cardiac variables (fixed effects: Ees, Ea, EDV and heart rate) associated with changes in LVEF. We also analyzed the relationship between LVEF with VAC and LVeff. Models were constructed using individual animals as subjects for random factors, and sequential experimental stages as repeated measurements. A Toeplitz covariance structure was selected based on the corrected Akaike's information criteria [21, 22]. Model parameters were estimated via the restricted maximum likelihood method and the estimated fixed effects quantified by the estimated value (95\% confidence interval). Linear regression analysis was used for determining the relationship between continuous variables. Fisher $z$ test was used for comparing correlations. Four-quadrant plots were used for assessing concordance between Ees and different LVEF calculations. Concordance was defined as the percentage of data in which the direction of change agreed. Excellent concordance was assumed when the concordance rate was $\geq 90 \%$. A $p$ value $<0.05$ was considered statistically significant. All statistical analyses were performed using MedCalc Statistical Software version 18.8 (MedCalc Software bvba, Ostend, Belgium; https://www. medcalc.org; 2016) and SPPS (SPSS 21, SPPS Inc, Chicago, IL).

\section{Results}

Evolution of main $L V$ variables during different experimental conditions A detailed description of the main changes in hemodynamics associated with the various interventions was previously reported [11]. Complementary data about changes in VAC, LVeff and diastolic function are reported in Tables 1 and 2.

Impact of global and diastolic function on LVEF The contribution of different cardiovascular variables on LVEF is detailed in Table 3. The main determinants of LVEF were Ees and Ea, and to a lesser extent EDV and heart rate. There was an inverse relationship between LVEF and Ea; therefore, for a given LV contractility state, a change in Ea will independently change LVEF in a reciprocal fashion. The association of EDV and heart rate to LVEF was minimal when compared with the prominent impact of Ees and Ea. Considering the estimate fixed effects shown in Table 2, the decrease in EDV during bleeding (from $234+50 \mathrm{ml}$ to $211 \pm 57 \mathrm{ml}$ ) would only explain a $3 \%$ in the observed LVEF increase after bleeding (from $49 \pm 11 \%$ to $55 \pm 13 \%$ ), while other

Table 1 Evolution of ventriculo-arterial coupling and left ventricular mechanical efficiency during different experimental stages

\begin{tabular}{llllll}
\hline & \multicolumn{2}{l}{ VAC (a.u.) } & & \multicolumn{2}{l}{ LV efficiency (\%) } \\
\cline { 2 - 3 } \cline { 5 - 6 } & Before & After & & Before & After \\
\hline $\begin{array}{llllll}\text { Afterload } \\
\text { Phenylephrine }\end{array}$ & $2.06 \pm 0.51$ & $2.36 \pm 0.51^{*}$ & $61 \pm 9$ & $55 \pm 10^{*}$ \\
Nitroprusside & $2.00 \pm 0.49$ & $1.42 \pm 0.44^{\dagger}$ & $61 \pm 10$ & $72 \pm 11^{\ddagger}$ \\
Preload & & & & \\
Bleeding & $2.02 \pm 0.61$ & $1.57 \pm 0.51^{*}$ & $58 \pm 11$ & $67 \pm 11^{\dagger}$ \\
Fluid bolus & $1.72 \pm 0.63$ & $2.06 \pm 0.73^{*}$ & $65 \pm 12$ & $57 \pm 12^{*}$ \\
Contractility & & & & \\
Esmolol & $1.50 \pm 0.36$ & $2.43 \pm 0.61^{\dagger}$ & $68 \pm 7$ & $47 \pm 3^{\ddagger}$ \\
Dobutamine & $1.71 \pm 0.47$ & $1.14 \pm 0.34^{\ddagger}$ & $65 \pm 7$ & $75 \pm 1^{\ddagger}$ \\
\hline
\end{tabular}

Data are presented as mean $\pm \mathrm{SD}$

A.u. arbitrary units, VAC ventriculo-arterial coupling, LVeff left ventricular mechanical efficiency

${ }^{*} p<0.05,{ }^{\dagger} p \leq 0.001,{ }^{\ddagger} p \leq 0.0001$ versus "before" stage 
Table 2 Evolution of left ventricular diastolic function variables

\begin{tabular}{|c|c|c|c|c|}
\hline & \multicolumn{2}{|c|}{$\tau(\mathrm{tau})(\mathrm{ms})$} & \multicolumn{2}{|c|}{ LV stiffness $(\beta)$} \\
\hline & Before & After & Before & After \\
\hline \multicolumn{5}{|l|}{ Afterload } \\
\hline $\begin{array}{c}\text { Phenyle- } \\
\text { phrine }\end{array}$ & $29.3 \pm 2.9$ & $33.5 \pm 3.24^{\dagger}$ & $0.013 \pm 0.003$ & $0.014 \pm 0.003^{*}$ \\
\hline Nitroprusside & $27.4 \pm 3.4$ & $22.4 \pm 2.2^{\ddagger}$ & $0.013 \pm 0.003$ & $0.012 \pm 0.003^{+}$ \\
\hline \multicolumn{5}{|l|}{ Preload } \\
\hline Bleeding & $27.5 \pm 3.5$ & $22.8 \pm 3.2^{\ddagger}$ & $0.012 \pm 0.003$ & $0.012 \pm 0.003$ \\
\hline Fluid bolus & $25.4 \pm 4.3$ & $28.9 \pm 4.1^{\dagger}$ & $0.012 \pm 0.003$ & $0.011 \pm 0.002^{*}$ \\
\hline \multicolumn{5}{|l|}{ Contractility } \\
\hline Esmolol & $27 \pm 3.8$ & $35 \pm 5.4^{*}$ & $0.013 \pm 0.002$ & $0.012 \pm 0.003$ \\
\hline Dobutamine & $27.1 \pm 4$ & $24.4 \pm 4.5^{*}$ & $0.012 \pm 0.002$ & $0.012 \pm 0.002$ \\
\hline
\end{tabular}

Data are presented as mean \pm SD

$\tau$ (tau) time constant of the isovolumetric LV pressure relaxation, $\beta$ LV chamber stiffness constant obtained from the end-diastolic pressure-volume relationship ${ }^{*} p<0.05,{ }^{\dagger} p \leq 0.001,{ }^{\ddagger} p \leq 0.0001$ versus before stage

Table 3 Estimated values of different variables on left ventricular ejection fraction (LVEF) according to a linear mixed-effects model analysis

\begin{tabular}{lclr}
\hline Fixed effects & Estimate & $\begin{array}{l}\text { 95\% confidence } \\
\text { interval }\end{array}$ & $\boldsymbol{p}$ value \\
\hline $\begin{array}{l}\text { Global cardiac function } \\
\text { Ees }\left(\mathrm{mmHg} \mathrm{m}^{-1}\right)\end{array}$ & 44.89 & 34.28 to 55.49 & $<0.001$ \\
Ea $\left(\mathrm{mmHg} \mathrm{ml}^{-1}\right)$ & -29.47 & -33.26 to -25.68 & $<0.001$ \\
LV EDV (ml) & -0.12 & -0.15 to -0.08 & $<0.001$ \\
Heart rate (bpm) & 0.17 & 0.05 to 0.29 & 0.006 \\
Diastolic function & & & \\
LV $\beta$ constant (a.u.) & 1340.3 & 474.9 to 1932.6 & $<0.001$ \\
T (ms) & -1.28 & -1.49 to -1.07 & $<0.001$ \\
VAC (Ea/Ees) (a.u.) & -10.6 & -11.85 to -9.37 & $<0.001$ \\
LV mechanical efficiency & 0.87 & 0.85 to 0.90 & $<0.001$ \\
(\%) & & & \\
\hline
\end{tabular}

Estimate reflects the average change in the LVEF per unit increase of each fixed effect

Ees left ventricular end-systolic elastance; $E a$ effective arterial elastance, $L V$ left ventricle, EDV end-diastolic volume, EDPVR slope of the end-diastolic pressurevolume relationship, $\beta \mathrm{LV}$ chamber stiffness constant obtained from the enddiastolic pressure-volume relationship, $\tau$ (tau) time constant of the isovolumetric $\mathrm{LV}$ pressure relaxation, VAC ventriculo-arterial coupling

factors such as a decreased Ea would also explain the overall LVEF increase. On the other hand, the increase in EDV after fluid administration (from $215 \pm 52 \mathrm{ml}$ to $259 \pm 47 \mathrm{ml}$ ) would explain the $5 \%$ decrease observed in LVEF (from $54 \pm 13 \%$ to $47 \pm 10 \%$ ), while a decrease in Ees and heart rate would also contribute to the net effect in LVEF after volume expansion. Moreover, for a relative $10 \%$ increase in Ees, Ea, EDV and heart rate, LVEF will change by $4.5 \%,-2.9 \%,-0.01 \%$ and $0.02 \%$, respectively. Even both active and passive diastolic function variables, $\tau$ and LV chamber stiffness constant $(\beta)$, had a significant relationship with LVEF, this was small considering the minor changes observed in diastolic function during the study. For example, for a change in LV stiffness constant of 0.001 , as seen during afterload interventions or during fluid administration, LVEF will alter by $1.3 \%$.

LVEF as an integrated index of cardiovascular performance LVEF was strongly related to VAC and LVeff (Table 2 and Fig. 1). A decrease in LVEF was related to a higher VAC and a lower LVeff. A decrease in 10\% in LVeff was associated with a parallel $9 \%$ reduction in LVEF.

Performance of an afterload-corrected EF for estimating Ees Given the predominant effect of Ea, we proposed an afterload-adjusted LVEF using a simple nonlinear approach for estimating LVEF:

$$
\mathrm{LVEF}_{\mathrm{EA}}=\mathrm{EF} \times \sqrt{\mathrm{Ea}},
$$

which it can be simplified as:

$$
\operatorname{LVEF}_{\mathrm{EA}}=\frac{\mathrm{SV}}{\mathrm{EDV}} \times \sqrt{\frac{\mathrm{Pes}}{\mathrm{SV}}} .
$$

Therefore,

$$
\mathrm{LVEF}_{\mathrm{EA}}=\sqrt{(\mathrm{Pes} \times \mathrm{SV})} / \mathrm{EDV} \text {. }
$$

Since LVEF mainly depends on LV contractility and afterload, this modification of the standard formula would consider both the direct relationship of LVEF with Ees and the inverse correlation with Ea. By using the square root function of $\mathrm{Ea}$, it would also take into account that the impact of afterload is greater at low levels of contractility [6]. Although we tested different potential linear and nonlinear approaches, we selected this adjustment based on the highest correlation with Ees $\left(r^{2}=0.75\right.$ vs $r^{2}=0.43$ for standard LVEF calculation; $p<0.0001$ for the comparison, Fig. 2), its simplicity and its clinical feasibility. This improvement still held even when Ea was estimated using radial MAP as surrogate for Pes (peripheral $\left.\mathrm{LVEF}_{\mathrm{EA}}=\sqrt{(\text { radial MAP } \times \mathrm{SV})} / \mathrm{EDV}\right)$ : $r^{2}=0.73$ ( $p=0.0001$ vs standard LVEF calculation). The combination of an afterload and preload adjustment did not improve this relationship $\left(r^{2}=0.73 ; p=0.6635\right.$ vs $\left.\mathrm{LVEF}_{\mathrm{EA}}\right)$. A simple approach that considered a linear relationship between Ea and $\operatorname{LVEF}\left(\mathrm{LVEF}_{\mathrm{EAlinear}}=\mathrm{EF} \times \mathrm{Ea}\right)$ also did not improve the relationship with Ees $\left(r^{2}=0.61\right.$; $p=0.0424$ vs $\left.^{2} \operatorname{LEF}_{\mathrm{EA}}\right)$.

Performance comparison for tracking changes in Ees during different experimental conditions Concordance analysis for different approaches for tracking Ees changes is shown in Fig. 3. While all three methods showed perfect tracking of Ees during contractility 


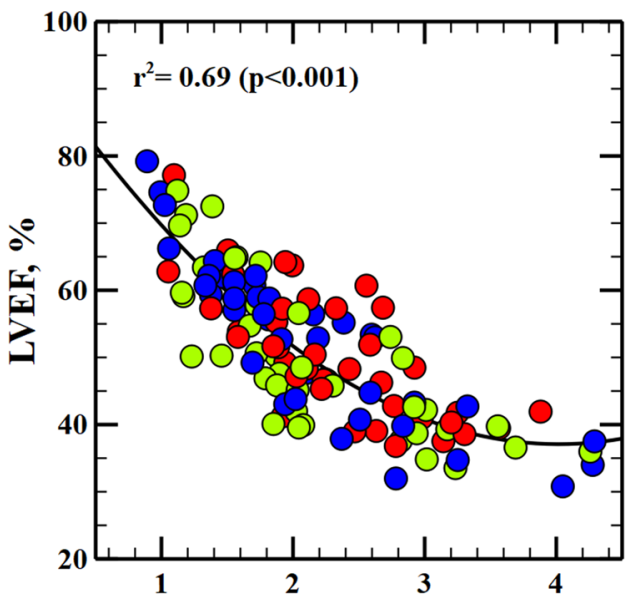

Ventriculo-arterial coupling, a.u. (Ea/Ees)

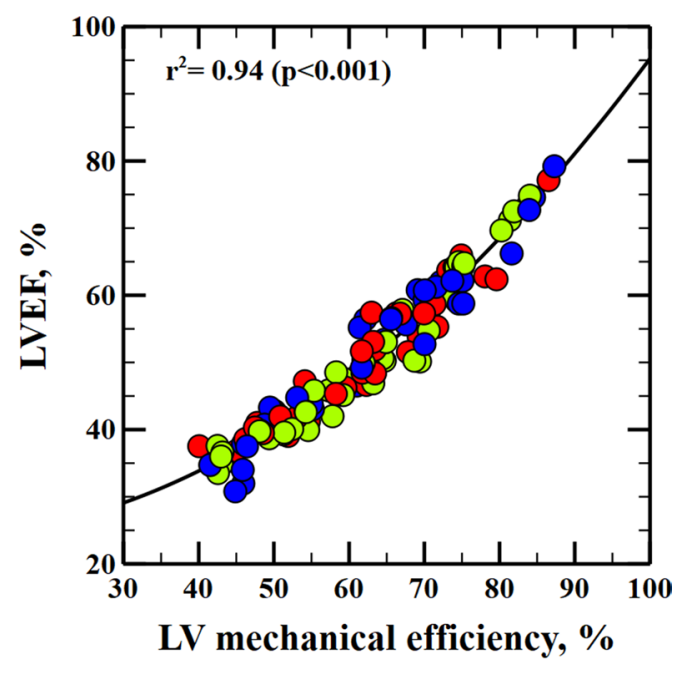

Fig. 1 Relationship between ventriculo-arterial coupling, left ventricular mechanical efficiency and left ventricular ejection fraction. Left: linear regression analysis between ventriculo-arterial coupling (VAC), calculated as the ratio between effective arterial elastance (Ea) and left ventricular end-systolic elastance (Ees), and left ventricular ejection fraction (LVEF). Right: linear regression analysis between left ventricular mechanical efficiency, calculated as the ratio between stroke work (SW) and the left ventricular pressure-volume area (PVA), and left ventricular ejection fraction. Colors inside circles represent different experimental interventions: red, afterload; green: preload; blue: contractility
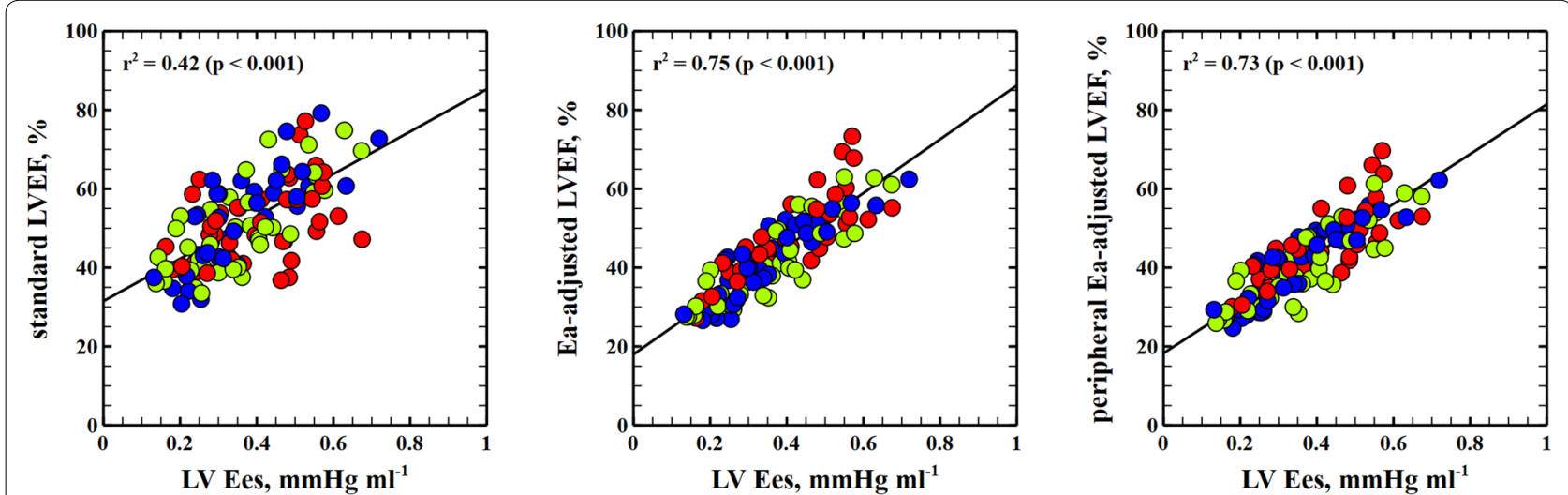

Fig. 2 Relationship between left ventricular end-systolic elastance (Ees) and standard left ventricular ejection fraction (LVEF) calculation, Ea-adjusted LVEF and peripheral Ea-adjusted LVEF. Linear regression analysis for left ventricular end-systolic elastance (Ees) and standard LVEF, LVEF corrected to effective arterial elastance (Ea = left ventricular end-systolic pressure/left ventricular stroke volume) and peripheral Ea-adjusted LVEF $\left(\mathrm{Ea}_{\text {periph }}=\right.$ radial mean arterial pressure/stroke volume). Colors inside circles represent different interventions: red, afterload; green: preload; blue: contractility

changes, when afterload was changed, standard LVEF poorly reflected Ees variations. On the other hand, both central and peripheral Ea-adjusted LVEF significantly improved the tracking of Ees during afterload interventions, while standard LVEF was better for tracking Ees changes during preload variations, although these changes were significantly smaller in amplitude compared to those observed during contractile and afterload variations.

\section{Discussion}

This study confirms that LV Ees and Ea are the main determinants of LVEF. Not surprisingly, we also corroborated the strong relationship between LVEF and both VAC and LVeff, which are mathematically coupled to Ees 

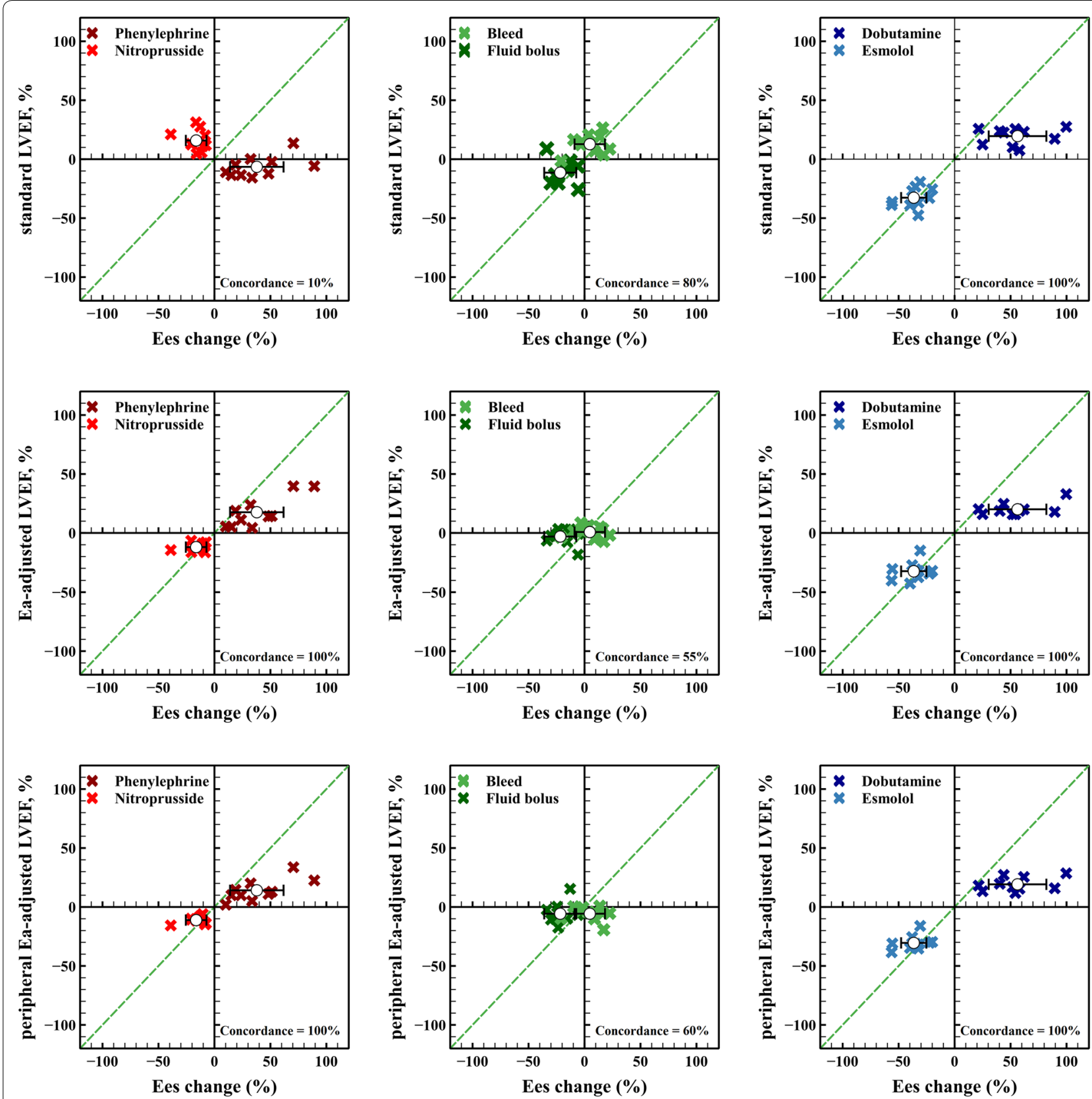

Fig. 3 Concordance analysis for percentage changes in different approaches for estimating left ventricular contractility and end-systolic elastance (Ees) during different experimental stages. Four-quadrant plots showing the relationship between percentage changes in left ventricular (LV) end-systolic elastance (Ees) and different approaches studied: standard LVEF calculation, LVEF adjusted to effective arterial elastance (Ea = left ventricular end-systolic pressure/stroke volume) and peripheral Ea-adjusted LVEF (Ea $a_{\text {periph }}=$ radial mean arterial pressure/stroke volume). Excellent trending capability was assumed when $\geq 90 \%$ of the data lie in the right-upper and the left-lower quadrants. Open circles with bars represent the mean percentage change on each stage. Dashed green lines represent the line of equality

and Ea. Thus, LVEF is mainly determined by the coupling between the LV systolic function and the arterial system and less so to changes in preload and heart rate. As such, LVEF represents more of a global index of cardiovascular performance rather than an absolute measure of LV contractility. We also propose an afterload correction to LVEF that significantly improved the performance over the standard LVEF calculation for estimating LV contractility during changes in loading conditions, while based on easily available parameters. 
Ideally, an index of contractility index should be sensitive to changes in myocardial contractile state but indifferent to loading conditions [6]. Although LVEF has been traditionally used as a clinical measure of LV systolic performance, its dependence on preload and especially afterload changes have been well documented experimentally over five decades ago by Krayenbuhl et al. [23], later corroborated in isolated canine ventricles by Kass et al. [24] and theoretically described by Robotham [6]. However, the recognition that LVEF does not only depend solely upon myocardial contractility but also upon other determinants of the ventricular function has been increasingly recognized in critically ill patients with the growing use of echocardiography in ICU to assess LV function $[3,9,25]$. For example, the afterload influence in LVEF has been particularly evident and documented in septic patients $[8,9,26]$. Impaired intrinsic contractility and vasoplegia are the hallmark of the hemodynamic disorders in septic shock $[27,28]$. Under these conditions, a normal LVEF may not necessarily indicate a normal LV contractility, but rather reflect the profoundly impaired LV afterload [9, 29]. Similarly, a low LVEF within the context of a decreased LV afterload would support the diagnosis of severely depressed LV systolic function. On the other hand, a reduction in LVEF after correcting arterial hypotension with vasopressors need not reflect a worsening of LV systolic function, but only the unmasking of existing LV dysfunction by normalization of LV afterload [10]. Only if LVEF still remains low after restoring arterial pressure and afterload, then an impairment in LV contractility is likely. This reasoning could explain the large variability of LV dysfunction reported in previous clinical studies and the lack of association between LVEF and mortality in septic shock $[8,30]$. As loading conditions can be significantly altered by vasopressors or fluid administration, differences on the timing and the amount of hemodynamic resuscitation could have influence the reported value of LVEF regardless the intrinsic contractile state. Accordingly, considering the multiple factors that can influence LVEF in septic shock patients, where perturbations of loading conditions are particularly frequent, the interpretation of LVEF as an index of LV contractility should be done cautiously and after considering the potential impact of these factors [25].

While afterload changes largely affected LVEF, preload variations, even when abrupt and of large amplitude, such as during bleeding and fluid loading, failed to greatly influence LVEF. This was particularly evident even if CO significantly increased during fluid loading (from $7.89 \pm 1.7 \mathrm{l} / \mathrm{min}$ to $9.11 \pm 2.42 \mathrm{l} / \mathrm{min} ; p=0.01$ ), while LVEF showed only a modest decrease (from $54 \pm 13 \%$ to $47 \pm 10 \% ; p=0.02$ ). This is presumably related to the fact that LVEF normalized changes in SV by EDV, while a reduction in LVEF after fluid infusion would be associated with an increase with EDV without a subsequent improvement in SV (non-preload dependency). Therefore, a decrease in LVEF after fluid administration could be indicative of a lack of preload responsiveness, while an unchanged or an increased LVEF would denote a significant increase in SV with volume administration (fluid responsiveness).

Additionally, LVEF is a function of both SV and EDV. Patients with sustained lusitropy but preserved SV will paradoxically have a reduced LVEF, owing to the increased EDV, but have better cardiovascular reserve. This assumption supports the multiple studies reporting a decreased LVEF in survivors of severe sepsis as compared to non-survivors [31]. Non-survivors presumably are unable to relax appropriately during diastole, while both survivors and non-survivors have sepsis-induced impaired systolic function. This hypothesis is supported by the association found between diastolic disfunction and mortality in septic patients [32,33]. As we did not also study the impact of sepsis in our porcine model, we cannot speculate further on this hypothesis. Moreover, the effects of our interventions on diastolic function, though measurable, were small and not relevant, because they minimally altered EDV. However, in the setting of altered diastolic function, as may occur in sepsis and LV hypertrophy, these effects may become relevant. Further focused investigation of these issues would be needed to address these concerns.

Our in vivo experimental study with an intact cardiovascular system confirms previous observations about the influence of the cardiac loading conditions on LVEF [23, 24, 34-36], but more importantly, it also provides the empirical demonstration about the real nature of LVEF. Since LVEF is primarily governed by the influence of Ees and Ea, it mainly reflects the balance between LV contractile function and the arterial system. Therefore, LVEF should be considered as a ventriculo-arterial coupling index and a variable mostly related to LV systolic mechanical efficiency, but not a pure measure of LV systolic performance $[6,37]$. Furthermore, even if LVEF is associated with a unique VAC and LV mechanical efficiency state, the same LVEF level can be obtained with different values of Ees and Ea. Thus, the clinical utility of measures of LVEF under conditions of varying cardiovascular states is unclear and may be misleading.

Thirty years ago Robotham stated that LVEF "reflects the integrated system's ability to cope, under the conditions at the time of measurement, with abnormalities in preload, afterload and/or contractility", and therefore, "ejection fraction becomes a measure, not of ventricular performance, but of the integrated system's performance in dealing with a pathological process" [6]. Although his 
measures were less accurate than ours, our data agree with his fundamental observation. Accordingly, an abnormal LVEF represents the inability of the cardiovascular system to modulate the current contractile and loading conditions for sustaining normal homeostasis. On the contrary, a normal LVEF does not necessarily mean that LV contractility is preserved, but it rather represents a composite variable expressing the cardiovascular system's response to the current loading and contractile conditions. Practically speaking, a low LVEF should always be considered as a sign of impaired cardiovascular function, reflecting an unfavorable LVeff and VAC, but it does not inform about the underlying mechanisms leading to its low value, which could be an impaired contractility, increased afterload or both. Furthermore, a normal LVEF, particularly in critically ill patients, should never be interpreted as evidence of normal LV systolic function, especially in the presence of hypotension and systemic hypoperfusion. Relevant to this last point, increased LVEF due to a predominant reduction on LV afterload, even in the presence of an impaired LV contractility, has been associated with a higher mortality $[8,38,39]$.

While standard LVEF remains of clinical interest, since it gives a global picture of LV pump function for the given contractility and loading conditions, we propose reporting an Ea-adjusted LVEF to significantly improve the ability of LVEF to tracking changes in Ees during acute perturbations of LV afterload, without affecting the performance during changes in contractility. Moreover, we have recently demonstrated that radial, femoral and aortic MAP values are interchangeably as valid surrogates for the estimation of Pes, so LVEF can be adjusted using a peripheral estimation of Ea regardless the arterial pressure measurement site [12]. Since the additional information required to calculate $\mathrm{LVEF}_{\mathrm{EA}}$ can be obtained using currently available measures of arterial pressure and ventricular function, its application for estimating LV contractility could be easily implemented. This afterloadcorrected LVEF could be particularly helpful for identifying those patients with depressed LV contractility in the setting of altered loading conditions, as seen during septic shock. Furthermore, identification of depressed contractility by the Ea-corrected LVEF in the context of a persistent tissue hypoperfusion could theoretically better define the population potentially eligible for inotropic therapy. However, the usefulness and superiority of these surrogates in the clinical practice needs further clinical validation.

Our results require a few comments. First, we did not use echocardiography for calculating LVEF, which is the standard in clinical practice. Instead, we used the data obtained from the LV PV analysis by the classical conductance catheter technique. This method has been demonstrated to provide the most comprehensive description of the LV function [40], whereas estimation of LVEF by standard echocardiography relies on geometrical assumptions and is prone to technical inaccuracies and inter-/intra-observer variability. Thus, echocardiographic estimates of LVEF should show even greater variance than our findings, underscoring further the lack of sensitivity of LVEF estimates to track LV contractility. However, our results are not limited to the use of this conductance catheter methodology, since they should remain valid to any estimation of LVEF, such as that obtained from the 3D echocardiography or global ejection fraction from transpulmonary thermodilution. However, as our LVEF estimation was based on the volumetric data from the conductance catheter, these clinical estimates of LVEF would still rely on the accuracy of their measurements and their assumptions about the LV geometry. Finally, our study was performed on pigs receiving anesthesia, so our results should be interpreted with caution when extrapolating to human cardiovascular physiology in awake individuals.

\section{Conclusions}

Changes in LVEF are mainly influenced by LV contractility and afterload and strongly related with ventriculoarterial coupling and LV mechanical efficiency. Therefore, LVEF primarily represents an integrated index of cardiovascular performance and LV mechanical efficiency rather than an actual measure of LV contractility. We proposed an afterload-adjusted LVEF, which significantly improved the ability to track LV contractility.

\section{Abbreviations}

LV: left ventricle; Ees: end-systolic elastance; LVeff: LV mechanical efficiency; LVEF: left ventricular ejection fraction; $L_{V E F}$ : left ventricular ejection fraction adjusted to effective arterial elastance; PV: pressure-volume; CO: cardiac output; IVC: inferior vena cava; Pes: left ventricular end-systolic pressure; SV: stroke volume; EDV: left ventricular end-diastolic volume; ESV: left ventricular endsystolic volume; Ea: effective arterial elastance; $E$ : elastance; $V_{0}$ : left ventricular unstressed volume; MAP: mean arterial pressure; AICc: Akaike's information criteria; ICU: intensive care unit.

\section{Authors' contributions}

MIMG, MRP and MC conceived the study. MIMG, ZJ and MRP studied the design. MIMG, ZJ, FH, CH performed experimental research. MIMG, ZJ, MRP and FH analyzed and interpreted the data. MIMG, ZJ, JJS and FH drafted the manuscript. All authors reviewed it, contributed significantly to its critical review and approved the final version of the manuscript. All authors ensure the accuracy or integrity of the results of this study and will be accountable for any question related with this work. All authors read and approved the final manuscript.

\section{Author details}

${ }^{1}$ Unidad de Cuidados Intensivos, Hospital Universitario SAS de Jerez, C/Circunvalación, s/n, 11407 Jerez de la Frontera, Spain. ${ }^{2}$ Edwards Lifesciences, Irvine, CA, USA. ${ }^{3}$ Orlando Regional Medical Center, Orlando Health, Orlando, FL, USA. ${ }^{4}$ Department Anaesthesia and Intensive Care Units, Humanitas Research Hospital, Humanitas University, Milan, Italy. ${ }^{5}$ Department of Critical Care Medicine, University of Pittsburgh School of Medicine, Pittsburgh, USA. 


\section{Acknowledgements}

We are grateful to all laboratory staff of the Edwards Research Center for their valuable assistance in this work.

\section{Competing interests}

MIMG is a consultant to Edwards Lifesciences and received honoraria and/or travel expenses from Deltex Medical. M.R. Pinsky is a consultant to Edwards LifeSciences, LiDCO Ltd., and Cheetah. MC has received honoraria and/or travel expenses from Edwards Lifesciences, LiDCO, Cheetah, Bmeye, Masimo and Deltex Medical. CH is a consultant to Edwards Lifesciences. ZJ, JJS and FH are Edwards Lifesciences employees.

\section{Availability of data and materials}

The data that support the findings of this study are available from the corresponding author upon reasonable request.

\section{Consent for publication}

Not applicable.

\section{Ethics approval and consent to participate}

The study was approved for the use of swine by the Institutional Animal Care and Use Committee (IACUC) at the Edwards Research Center, and all experimentation was performed in accordance with the USDA Animal Welfare Act regulations (AWArs) and the Guide for the Care and Use of Laboratory Animals (ILAR, NAP, Washington, DC, 2010, 8th edition). The Test Facility is accredited by the Association for the Assessment and Accreditation of Laboratory Animal Care, International (AAALACi) and registered with the United States Department of Agriculture to conduct research with laboratory animals.

\section{Funding}

Edwards Lifesciences provided the software, hardware and animals for the study.

\section{Publisher's Note}

Springer Nature remains neutral with regard to jurisdictional claims in published maps and institutional affiliations.

Received: 7 January 2019 Accepted: 11 April 2019 Published online: 16 April 2019

\section{References}

1. Weir RA, McMurray JJ, Velazquez EJ. Epidemiology of heart failure and left ventricular systolic dysfunction after acute myocardial infarction: prevalence, clinical characteristics, and prognostic importance. Am J Cardiol. 2006;97(10A):13F-25F.

2. Curtis JP, Sokol SI, Wang Y, Rathore SS, Ko DT, Jadbabaie F, et al. The association of left ventricular ejection fraction, mortality, and cause of death in stable outpatients with heart failure. J Am Coll Cardiol. 2003;42(4):736-42.

3. Cikes M, Solomon SD. Beyond ejection fraction: an integrative approach for assessment of cardiac structure and function in heart failure. Eur Heart J. 2016;37(21):1642-50.

4. Kim EK, Cho J, Kim JY, Chang SA, Park SJ, Choi JO, et al. Early decline in left ventricular ejection fraction can predict trastuzumab-related cardiotoxicity in patients with breast cancer: a study using 13 years of registry data. Cancer Res Treat. 2019;51(2):727-36.

5. Brignole M, Auricchio A, Baron-Esquivias G, Bordachar P, Boriani G, Breithardt OA, et al. 2013 ESC Guidelines on cardiac pacing and cardiac resynchronization therapy: the Task Force on cardiac pacing and resynchronization therapy of the European Society of Cardiology (ESC). Developed in collaboration with the European Heart Rhythm Association (EHRA). Eur Heart J. 2013;34(29):2281-329.

6. Robotham JL, Takata M, Berman M, Harasawa Y. Ejection fraction revisited Anesthesiology. 1991;74(1):172-83.

7. Konstam MA, Abboud FM. Ejection fraction: misunderstood and overrated (changing the paradigm in categorizing heart failure). Circulation. 2017;135(8):717-9.
8. Boissier F, Razazi K, Seemann A, Bedet A, Thille AW, de Prost N, et al. Left ventricular systolic dysfunction during septic shock: the role of loading conditions. Intensive Care Med. 2017;43(5):633-42.

9. Repesse X, Charron C, Vieillard-Baron A. Evaluation of left ventricular systolic function revisited in septic shock. Crit Care. 2013;17(4):164.

10. Vieillard-Baron A, Caille V, Charron C, Belliard G, Page B, Jardin F. Actual incidence of global left ventricular hypokinesia in adult septic shock. Crit Care Med. 2008;36(6):1701-6.

11. Monge Garcia MI, Jian Z, Settels JJ, Hunley C, Cecconi M, Hatib F, et al. Performance comparison of ventricular and arterial $\mathrm{dP} / \mathrm{dt}_{\max }$ for assessing left ventricular systolic function during different experimental loading and contractile conditions. Crit Care. 2018;22(1):325.

12. Monge Garcia MI, Jian Z, Settels JJ, Hatib F, Cecconi M, Pinsky MR. Reliability of effective arterial elastance using peripheral arterial pressure as surrogate for left ventricular end-systolic pressure. J Clin Monit Comput. 2018; . https://doi.org/10.1007/s10877-018-0236-y.

13. Kass DA, Yamazaki T, Burkhoff D, Maughan WL, Sagawa K. Determination of left ventricular end-systolic pressure-volume relationships by the conductance (volume) catheter technique. Circulation. 1986;73(3):586-95.

14. Baan J, van der Velde ET, de Bruin HG, Smeenk GJ, Koops J, van Dijk AD, et al. Continuous measurement of left ventricular volume in animals and humans by conductance catheter. Circulation. 1984;70(5):812-23.

15. Suga H, Sagawa K, Shoukas AA. Load independence of the instantaneous pressure-volume ratio of the canine left ventricle and effects of epinephrine and heart rate on the ratio. Circ Res. 1973;32(3):314-22.

16. Sunagawa K, Sagawa K, Maughan WL. Ventricular interaction with the loading system. Ann Biomed Eng. 1984;12(2):163-89.

17. Sunagawa K, Maughan WL, Burkhoff D, Sagawa K. Left ventricular interaction with arterial load studied in isolated canine ventricle. Am J Physiol. 1983;245(5 Pt 1):H773-80.

18. Burkhoff D, Sagawa K. Ventricular efficiency predicted by an analytical model. Am J Physiol. 1986;250(6 Pt 2):R1021-7.

19. Mirsky I. Assessment of diastolic function: suggested methods and future considerations. Circulation. 1984;69(4):836-41.

20. Burkhoff D, Mirsky I, Suga H. Assessment of systolic and diastolic ventricular properties via pressure-volume analysis: a guide for clinical, translational, and basic researchers. Am J Physiol Heart Circ Physiol. 2005:289(2):H501-12

21. Fitzmaurice GM, Laird NM, Ware JH. Modeling the covariance. Applied longitudinal analyss. 2nd ed. Hoboken, NJ: Wiley; 2011. p. 165-88.

22. Brown H, Prescott R. Repeated measures data. Applied mixed models in medicine. 2nd ed. Chichester: Wiley; 2006. p. 215-70.

23. Krayenbuhl HP, Bussmann WD, Turina M, Luthy E. Is the ejection fraction an index of myocardial contractility? Cardiologia. 1968;53(1):1-10.

24. Kass DA, Maughan WL, Guo ZM, Kono A, Sunagawa K, Sagawa K. Comparative influence of load versus inotropic states on indexes of ventricular contractility: experimental and theoretical analysis based on pressurevolume relationships. Circulation. 1987;76(6):1422-36.

25. Cecconi M, De Backer D, Antonelli M, Beale R, Bakker J, Hofer C, et al. Consensus on circulatory shock and hemodynamic monitoring. Task force of the European Society of Intensive Care Medicine. Intensive Care Med. 2014;40(12):1795-815.

26. Parker MM, Shelhamer JH, Bacharach SL, Green MV, Natanson C, Frederick $\mathrm{TM}$, et al. Profound but reversible myocardial depression in patients with septic shock. Ann Intern Med. 1984;100(4):483-90.

27. Guarracino F, Ferro B, Morelli A, Bertini P, Baldassarri R, Pinsky MR. Ventriculoarterial decoupling in human septic shock. Crit Care. 2014;18(2):R80.

28. Barraud D, Faivre V, Damy T, Welschbillig S, Gayat E, Heymes C, et al. Levosimendan restores both systolic and diastolic cardiac performance in lipopolysaccharide-treated rabbits: comparison with dobutamine and milrinone. Crit Care Med. 2007;35(5):1376-82.

29. Vieillard-Baron A. Septic cardiomyopathy. Ann Intensive Care. 2011;1(1):6.

30. Huang SJ, Nalos M, McLean AS. Is early ventricular dysfunction or dilatation associated with lower mortality rate in adult severe sepsis and septic shock? A meta-analysis. Crit Care. 2013;17(3):R96.

31. Landesberg G, Gilon D, Meroz Y, Georgieva M, Levin PD, Goodman S, et al. Diastolic dysfunction and mortality in severe sepsis and septic shock. Eur Heart J. 2012;33(7):895-903.

32. Sanfilippo F, Corredor C, Arcadipane A, Landesberg G, Vieillard-Baron A, Cecconi $M$, et al. Tissue Doppler assessment of diastolic function and 
relationship with mortality in critically ill septic patients: a systematic review and meta-analysis. Br J Anaesth. 2017;119(4):583-94.

33. Sanfilippo F, Corredor C, Fletcher N, Landesberg G, Benedetto U, Foex P, et al. Diastolic dysfunction and mortality in septic patients: a systematic review and meta-analysis. Intensive Care Med. 2015;41(6):1004-13.

34. Nixon JV, Murray RG, Leonard PD, Mitchell JH, Blomqvist CG. Effect of large variations in preload on left ventricular performance characteristics in normal subjects. Circulation. 1982;65(4):698-703.

35. Quinones MA, Gaasch WH, Alexander JK. Influence of acute changes in preload, afterload, contractile state and heart rate on ejection and isovolumic indices of myocardial contractility in man. Circulation. 1976;53(2):293-302.

36. Tsakiris AG, Rusell A, Vanderberg MB, Banchero N, Sturm RE, Wood EH. Variations of left ventricular end-diastolic pressure, volume, and ejection fraction with changes in outflow resistance in anesthetized intact dogs. Circ Res. 1968;23:213-22.

37. Sunagawa K, Maughan WL, Sagawa K. Optimal arterial resistance for the maximal stroke work studied in isolated canine left ventricle. Circ Res. 1985;56(4):586-95.

38. Paonessa JR, Brennan T, Pimentel M, Steinhaus D, Feng M, Celi LA. Hyperdynamic left ventricular ejection fraction in the intensive care unit. Crit Care. 2015;19:288.

39. Weng L, Liu YT, Du B, Zhou JF, Guo XX, Peng JM, et al. The prognostic value of left ventricular systolic function measured by tissue Doppler imaging in septic shock. Crit Care. 2012;16(3):R71.

40. Kass DA. Clinical evaluation of left heart function by conductance catheter technique. Eur Heart J. 1992;13 Suppl E:57-64.

\section{Submit your manuscript to a SpringerOpen ${ }^{\odot}$ journal and benefit from:}

- Convenient online submission

- Rigorous peer review

- Open access: articles freely available online

- High visibility within the field

- Retaining the copyright to your article

Submit your next manuscript at $\boldsymbol{\nabla}$ springeropen.com 